\title{
Lingue in contatto: un caso di prestigio linguistico
}

\author{
Mila Samardžić \\ Università di Belgrado, Facoltà di filologia \\ milasamardzic@fil.bg.ac.rs
}

Jezikiv stiku: primer jezikovnega prestiža

Predmet prispevka je pregled vpliva italijanskega jezika (in beneškega narečja) na knjižni srbski jezik ter na lokalne govore. Ti vplivi segajo v srednji vek in takorekoč brez premora trajajo do današnjih dni. Cilj prispevka je pokazati, kako se je, zahvaljujoč družbeno-ekonomskim in kulturnim okoliščinam, italijanščina vsilila kot prestižni jezik v odnosu do srbščine in kako je odnos med tema dvema jezikoma v stoletjih potekal načeloma enosmerno.

Ključne besede: jezikovno izposojanje, kontaktna lingvistika, italijanščina, srbščina, jezikovni prestiž

Languages in contact: a case of linguistic prestige

The article aims to offer a review of the influences exerted by the Italian language (and the Venetian dialect) on the Serbian literary language as well as on the local dialects. These impacts date back to the Middle Ages and, in practice uninterruptedly, persist to the present day. The aim of the paper is to demonstrate how, due to socio-economic and cultural circumstances, Italian has been able to establish itself as a prestigious language compared to Serbian and how the relationship between the two languages over the centuries has always been essentially monodirectional.

Keywords: Language loans, Contact Linguistics, Italian, Serbian, Linguistic Prestige

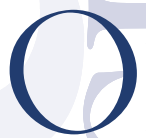

ltre all'interferenza e alla commutazione del codice, uno dei fenomeni linguistici del contatto sono i prestiti. I prestiti sostanzialmente riguardano il materiale di superficie, in primo luogo gli elementi lessicali, parole, che passano da una lingua a un'altra. Dunque, il contatto interessa maggiormente il sistema lessicale mentre per gli influssi riguardanti altri livelli linguistici (sintassi o fonologia) ci vogliono secoli. È un fenomeno dovuto ai fattori extralinguistici: le lingue possono entrare in contatto per contiguità territoriale o per via di vicende politiche, migrazioni, guerre, scambi culturali e commerciali. "È quindi ovvio che il passaggio di parole da una lingua all'altra sarà tanto più facile quanto più stret- ti saranno i rapporti fra le popolazioni parlanti quelle lingue" (Zolli 1976, I). In questo senso è sempre stato importante il fenomeno di prestigio (Berruto 1997, 90): la superiorità culturale, politica, economica o tecnologica di una cultura provoca l'accoglimento del materiale lessicale della lingua di quel popolo in altre lingue. Nel nostro lavoro sarà esaminato un caso di contatto asimmetrico (o verticale) perché le due lingue, durante secoli di diffuso e molteplice rapporto, hanno sempre avuto differente prestigio socioeconomico e culturale e una delle due lingue ha sempre avuto una posizione dominante rispetto all'altra. La lingua fonte (nel nostro caso l'italiano) ha sempre esercitato influssi sulla lingua ricevente (il serbo) creando un rapporto, come 
avremo modo di concludere, (quasi) esclusivamente unidirezionale.

\section{Influssi italiani nel passato}

Essendo una delle grandi lingue di cultura, l'italiano ha sempre esportato parole: sia nel passato che nel presente. Ha espresso la civiltà che ha dato un cospicuo contributo alla formazione della cultura occidentale e che in varie epoche è stata un punto di riferimento per le arti figurative, musica, letteratura (Serianni 2017). L'italiano ha esercitato influssi lessicali in vari periodi storici. E non solo l'italiano ma anche i suoi dialetti, soprattutto il dialetto veneziano. Fino a tutto il Cinquecento l'italiano e i volgari italiani hanno quella posizione centrale nella letteratura europea e fra il Cinque e l'Ottocento, insieme ad alcuni suoi dialetti, hanno partecipato alla formazione della lingua franca mediterranea:

$$
\begin{aligned}
& \text { Gli italianismi riflettono i contatti, imme- } \\
& \text { diati o mediati, con la civiltà italiana e varia- } \\
& \text { no a seconda delle circostanze. Dopo che } \\
& \text { nell'alto medioevo il Mediterraneo era sta- } \\
& \text { to dominato dalle flotte bizantine e poi sa- } \\
& \text { racene, dal rooo, e soprattutto con le crocia- } \\
& \text { te, emersero prima Amalf, poi Pisa, Genova } \\
& \text { e Venezia, che assursero a potenze navali do- } \\
& \text { minanti. Non sorprende che l'influsso di } \\
& \text { Venezia e del veneziano fosse più forte nel } \\
& \text { Levante, quello di Genova e del genovese } \\
& \text { nel Ponente, e che gli italianismi marinare- } \\
& \text { schi passati nelle lingue romanze occidentali } \\
& \text { erano più genovesi ( } 88 \text { di numero) che vene- } \\
& \text { ziani (44), mentre quelli passati nelle lingue } \\
& \text { balcaniche e nell'arabo erano più veneziani } \\
& \text { (47) che genovesi (3) (Stammerjohann } 20 \text { Io). }
\end{aligned}
$$

Dopo un periodo di stagno, il contributo decisivo al prestigio dell'italiano nel mondo, a partire dalla seconda metà del Seicento, viene dalla grande fortuna della musica e dell'opera italiana: l'italiano dei libretti dell'opera finisce per diventare una specie di esperanto del mondo musicale internazionale. Più recentemente, il prestigio dell' italiano è legato al suo successo internazionale negli ambiti legati al fenomeno di made in Italy: nella moda, nella gastronomia, nel cinema, nello stile di vita. Molte parole italiane appartenenti a queste varie sfere sono entrate (adattate o meno) in altre lingue e appartengono ormai al lessico internazionale: "Le strade più eleganti di tutte le metropoli del mondo sono gremite di insegne con nomi italiani, sono italiani la gran parte dei più noti stilisti, è la moda italiana a imporsi come la più sofisticata ed elegante, è il design italiano il più ricercato e imitato" (Bertini Malgarini 1994, 891). Questo è il caso, per esempio, di parole come pizza, espresso, dolcevita, tiramisü, macchina ecc. Torneremo nella seconda parte del lavoro ai prestiti italiani diffusi nell'età contemporanea, e per il momento ricordiamo come l'italiano nei secoli passati abbia lasciato tracce profonde nel lessico legato alla religione (papa, monsignore, suora, (frate) cappuccino; cfr. Rossi e Wank 2010), all'abbigliamento (cappa, mantelletta, mozzetta, sottana, stola; cfr. D’Achille e Patota 2016), agli sport e ai giochi (maneggio, lotteria, palio, scudetto, tarocco, tombola; cfr. Arcangeli 2007) e alla scienza (malaria, pellagra, tarantismo, neutrino; cfr. Casapullo 2009).

\section{Influssi italiani in serbo}

$$
\begin{aligned}
& \text { I paesi balcanici e quelli arabi intorno al Me- } \\
& \text { diterraneo }[. . .] \text { sono legati all'Italia da una } \\
& \text { 'trama' culturale e commerciale che a vol- } \\
& \text { te è di tradizione secolare, a volte è rinverdi- } \\
& \text { ta dalla recezione dei moderni canali televi- } \\
& \text { sivi italiani e che in ogni caso è ancora tutta } \\
& \text { da documentare e valorizzare (Bettoni I993, } \\
& \text { 455-456). }
\end{aligned}
$$

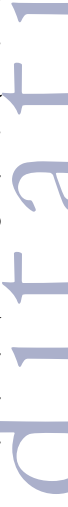

La lingua italiana ha esercitato in vari modi, in varie epoche, in varie sfere e per vari motivi influssi sul lessico delle parlate serbe contribuendo al loro arricchimento. Nella lingua serba se è registrato un numero cospicuo di italianismi. Questo grazie anche agli strettissimi e diversissimi contatti fra le due sponde dell'Adriatico che durano da secoli e che si rispecchiano particolarmente nelle parlate e dialetti slavi della costa adriatica. Infatti l'italiano ha costituito una 
fonte preziosa per il riempimento delle lacune (particolarmente nei settori tecnici e scientifici) presentatesi nei tempi della formazione e della standardizzazione della lingua letteraria serba. Inoltre, è registrato un numero cospicuo di italianismi (e venezianismi) nelle parlate croate dell'Adriatico orientale: una parte ha mantenuto lo statuto dialettale mentre un'altra, non meno rilevante, è finita nella varietà standard. Infine, abbiamo registrato anche un gruppo di parole che, grazie al dominio della cultura italiana in alcune discipline artistiche o scientifiche, oggi fanno parte del patrimonio lessicale europeo.

La rassegna che offriamo non sarà esaustiva bensì cercherà di offrire un quadro illustrativo del fenomeno. Verranno presi in considerazione sia gli italianismi adattati (di epoche più remote) che gli italianismi integrali (risalenti ai tempi più recenti). Attraverso i cambiamenti nella traslitterazione si potrà valutare il grado di adattamento alle regole fonomorfologiche della lingua ricevente: è comune infatti l'adattamento dei prestiti più antichi, mentre i più recenti mantengono la grafia dell'originale (con parziali adattamenti nella pronuncia) anche se si registrano nella contemporaneità moltissime oscillazioni. Non saranno trascurati i cosiddetti pseudoitalianismi, forme che sembrano italiane ma non lo sono (come tuttifrutti), i deonomastici (come paparazzo), i nomi derivati da toponomastici o etnici (come bologna che vale un "tipo di salsiccia") e i marchionimi (che testimoniano la presenza dei referenti nella cultura d'arrivo al punto da far smarrire nella coscienza dell'utente l'originaria dipendenza dal marchio stesso: è il caso di Jacuzzi). Presenteremo anche i casi di italianismi che nel passaggio al serbo hanno subito uno slittamento semantico (come confetti che non mantiene il significato di "piccolo dolce costituito da una mandorla, una nocciola o un pistacchio rivestiti di zucchero" - ma, come in alcune altre lingue europee - spagnolo, catalano o francese - assume il significato di "coriandoli"; o limonata che, oltre al significato originario, ne introduce un altro di "opera artistica dolciastra, senza contenuti profondi"). Sarà interessante individuare la produttività delle forme, segno indiscutibile dell'acclimazione della voce nella lingua d'arrivo. Sono quelli che Serianni (2017) definisce "italianismi di secondo grado": si prenderanno in considerazione le voci derivate da italianismi (da trippa in serbo tripica). Infine, offriremo dati relativi al genere (che può essere modificato o assegnato al neutro, inesistente in italiano) e alla categoria grammaticale (cfr. gli esempi come bravo! e basta! che diventano forme invariabili appartenenti alla categoria di interiezioni).

La panoramica della presenza degli italianismi in serbo è organizzata cronologicamente e raggruppata in tre categorie: italianismi tradizionali (venezianismi e toscanismi presenti nella lingua letteraria e nei dialetti), italianismi culturali (i cosiddetti europeismi, presenti in diverse lingue europee) e italianismi nuovi che risalgono agli ultimi decenni (a partire dagli anni sessanta dello scorso secolo quando si è avuta una nuova ondata di italianismi che ha investito non solo la varietà standard ma anche le variabili diastratiche, specialmente il parlato giovanile, i linguaggi degli affari, sport, gastronomia, moda ecc. (Lipovac-Radulović 198I e 2009; Musić 1972, AA.VV. 2007; Klajn e Šipka 2008).

\section{Italianismi tradizionali e venezianismi \\ Quadro diacronico del fenomeno}

Di tutti i prestiti provenienti dalle lingue romanze in serbo gli italianismi sono l'esempio più importante del contatto diretto fra le due lingue. $\mathrm{Ci}$ rientra anche un cospicuo numero di venezianismi. Quando e in quali circostanze storiche e culturali gli italianismi cominciano a entrare nelle parlate slave? Le prime tracce dei prestiti romanzi, presenti nelle parlate del basso Adriatico orientale ma anche in altre zone slavofone, derivano dal latino volgare dell'area balcanica (račun, dal lat. Rationem, o košulja, dal lat. casula). La successiva ondata della penetrazione dei romanismi rientra nel cosiddetto "periodo dalmata". Tuttavia il flusso più notevole che ha colpito l'Adriatico orientale comincia a verificarsi a partire dal XIII secolo e si intensifica con la do- 
minazione veneziana nei secoli successivi. Si tratta per lo più di toscanismi e venezianismi entrati a far parte delle parlate urbane della costa (molto di meno di quelle rurali, del retroterra). Per molti casi, particolarmente quelli risalenti al XIII secolo, è difficile stabilire se i prestiti siano veneziani o toscani. A Ragusa, per esempio, le lingue della cultura sono latino, toscano e slavo, mentre la lingua del commercio nel XIII e nel XIV secolo è il veneziano. Il problema di stabilire l'origine precisa del prestito sta nel fatto che anche nelle stesse zone in una parola si riscontrano sia le varianti toscane che quelle veneziane. $\mathrm{Ma}$ in questa sede questo problema lo possiamo tralasciare perché non ci interessano tanto particolari strettamente linguistici quanto quelli culturali, di contatto (Musić 1972, 39).

È importante sottolineare che a partire dal XIII secolo gli influssi del toscano e del veneziano sono costanti (non occasionali) e diffusi e, praticamente, non smettono mai di verificarsi. E proprio al periodo dal XIII al XIX secolo risalgono molti esempi di venezianismi e toscanismi che hanno lasciato tracce sia nelle parlate locali (della costa) sia nella lingua letteraria.

Verso la metà dell'Ottocento il serbo ha avuto la riforma linguistica. La base della lingua letteraria in creazione era costituita dal dialetto erzegovese neostocavo. Ma quella lingua era lingua rurale, priva di terminologie tecniche e scientifiche, priva di nozioni astratte ed era necessario che quelle lacune fossero riempite di elementi stranieri. Proprio quei prestiti che all'epoca sono entrati a far parte di quella lingua riformata sono rimasti ancora oggi nel serbo standard. E naturalmente ne fanno parte anche romanismi arrivati nell'aria balcanica dalle coste dell'Adriatico orientale. Sono esempi di cosiddetti italianismi diretti, provenienti dai contatti diretti fra due (o più) lingue (e culture). Altri esempi sono arrivati tramite il tedesco, grazie alla "mediazione" dei fortissimi influssi austriaci sui Balcani. Gli italianismi che a partire dalla metà dell'Ottocento sono entrati a far parte del serbo letterario riguardano per lo più discipline in cui l'italiano nel Cinque e nel Seicento era dominante in tut- ta Europa. I più numerosi sono quelli della terminologia commerciale e bancaria (banka, konto, bilans, kredit, bankrotirati), musicale (tenor, bas, bariton, viola, kantata, sonata, serenada, duet, kvartet ecc.), militare (alarm, bataljon, kaplar, citadela, major, kapetan, general ecc), del teatro, dell'architettura ecc. (Klajn 1996, 46). Sono cosiddetti italianismi culturali chiamati anche europeismi ai quali torneremo successivamente. Adesso invece ci focalizziamo sui fenomeni locali, che riguardano parlate locali, dialettali, quelle dell'Adriatico orientale.

Dunque facciamo una breve rassegna di influssi italiani, sia della lingua letteraria che del dialetto veneziano. Chiaramente gli italianismi sono presenti piuttosto nella lingua letteraria e i toscanismi di regola si usavano più nella comunicazione formale il che è testimoniato da documenti rintracciabili negli archivi storici sparsi sulla costa orientale dell'Adriatico.

\section{I venezianismi}

Sono presenti nella lingua letteraria: barbun (ven. barbon, it. triglia); boca (ven. bozza, it. bottiglia); bura (ven. it. bora); datula (ven. datolo, it. dattero); dužd (ven., it. doge); gondola (ven., it. gondola); regata (ven., it. regata); salata (ven. salata, it. insalata).

Tuttavia sono molto più numerosi nelle parlate e nei dialetti dell'Adriatico orientale: balota (ven. balota, it. ballotta); batana (ven. battana); bevanda (ven. bevanda, it. vino rosso allungato); beškot (ven. bescoto, it. biscotto); bonaca (ven. bonazza, it. bonaccia); buraška (ven. borasca, it. burrasca); cotav (ven. zoto, it. zoppo); cukar (ven. zucaro, it. zucchero); ćakulati (ven. ciacolar, it. chiacchierare); dešperati se (ven. desperar, it. disperarsi); fritula (ven. fritola, it. frittella); furešt (ven. foresto, it. forestiero); gradela (ven. gradela, it. graticola, griglia); kamara (ven. camara, it. camera); kantun (ven. canton, it. canto); komodati se (ven. comodarse, it. accomodarsi); kužina (ven. cusina, it. cucina); lemozina (ven. lemosina, it. elemosina); marenda (ven. marenda, it. merenda); nevera (ven. nevera, it. tempesta); orada (ven. orada, it. orata); panceta (ven. panzeta, it. 
pancetta); pantegana (ven. pantegana, it. topo di fogna); pirun (ven. piròn, it. forchetta); skalina$d a$ (ven. scalinada; skkura ven. scuro, it. persiana); škver (ven. squero, it. cantiere); šempio (ven. sempio, it. scempio); tinel (ven. tinelo, it. tinello; cfr. Musić 1972).

\section{Italianismi diretti: $i$ toscanismi}

Anche i cosiddetti italianismi diretti o i toscanismi (Klajn 1996, 45-64) sono presenti nella lingua standard: agrumi, apetit (it. appetito), bakalar (it. baccalà < sp. bacalao < olandese ant. kabeljauw), barka (it. barca), bestija (it. bestia), faliti (it. fallire, nel significato di 'mancare' o 'venire meno'), fin (it. fino, fine), fontana, graciozan (it. grazioso), kaca (it. cazza), kancona (it. canzone), kapara, (it. caparra), kapetan (it. capitano), karabinjer (it. carabiniere), kavaljer (it. cavaliere), kredenac (it. credenza, mobile), kvintal (it. quintale < sp. quintal), macola (it. mazza, mazzuola), mafija (it. mafia), manjkati (it. mancare), milja (it. miglio), palma, pijaca (it. piazza, nel significato di 'mercato'), roba, ruzmarin (it. rosmarino), sardela (it. sarda, sardella), sardina, skala (it. scala), škrinja (it. scrigno), tombola, žbir (it. sbirro).

$\mathrm{Ci}$ si potrebbero aggiungere alcuni esempi di carattere regionale (rilevati nelle regioni litoranee, in Dalmazia, a Ragusa e nelle Bocche di Cattaro), come adio (it. addio), brodet (it. brodetto), fakin (ar. it. facchino), fešta (it. festa), $k a$ lamar (it. calamaro), kontati (it. contare), krepati (it. crepare), nona (it. nonna), nono (it. nonno), palenta (it. polenta), pašta (it. pasta), paštašuta (it. pastasciutta), piturati (it. pitturare), pjat (it. piatto), polpeta (it. polpetta), prošek (it. prosecco), riva (it. riva), rovinati (it. rovinare), siroko/silok (ar. it. scirocco), spaga (it. spago) ecc.

\section{Italianismi indiretti}

Altri prestiti, come milion (it. milione, fr. million, ted. Million), par (it. paio, ted. Paar), policija (it. polizia, fr. police, ted. Polizei), pomoranď̃a (it. pomarancia, arancia, ted. Pomeranze), ecc. probabilmente sono arrivati in serbo per via indiretta, cioè sono passati dall'italiano al serbo tramite la lingua tedesca o, meno frequentemente, quella francese (Klajn 1996, 49). Spesso la lingua mediatrice ha generato qualche spostamento semantico e mutamento fonetico o morfologico. Vediamo prima alcuni esempi nei quali riscontriamo alcuni dei cambiamenti fonetici nella pronuncia o quelli morfologici riguardo al genere dei sostantivi: korzo (korso, it. corso) secondo il tedesco viene pronunciato con la "s" sonora, parmezan (parmesan, it. parmigiano) conformemente al tedesco Parmesan e porcelan (porzelan, it. porcellana) in base al ted. Porzellan. Il sostantivo lak (it. lacca) è di genere maschile (cfr. ted. Lack), skica (it. schizzo) è di genere femminile (cfr. ted. f. Skizze), špijun (it. spia) è di genere maschile (cfr. ted. Spion), violina (it. violino) è di genere femminile (cfr. ted. f. Violine).

\section{Spostamenti del significato}

Negli esempi che seguono si verifica invece lo slittamento del significato della parola, spesso direttamente sotto l'influsso del tedesco: bruto (< it. brutto si usa nel significato di "(peso) lordo"), kantina (< it. cantina si usa nel significato di "spaccio nelle caserme"), parola (< it. parola si usa nel significato di "parola d'ordine"), tempo (< it. tempo si usa nel significato di "ritmo"), salutirati (< it. salutare si usa nel linguaggio militare con il significato di rendere onore $<$ ted. salutieren), sekirati (< it. seccare si usa nel significato di "preoccuparsi, angosciarsi", secondo il ted. sekkieren), štrapac e strapacirati (< it. strapazzo e strapazzare, con il significato di "cammino, lavoro faticoso", secondo ted. strapaze, strapazieren), ecc.

\section{Italianismi culturali (europeismi)}

Inoltre, esistono gli italianismi culturali che di regola sono europeismi: arsenal (it. ven. arsenale, originariamente parola araba), bagatela (it. bagattella), balet (it. balletto), balerina (it. ballerina), banka (it. banca), bizaran (it. bizzarro), civil (it. civile), diletant (it. dilettante), fašizam (it. fascismo), fasista (it. fascista), figura, forma, galop (it. galoppo), galopirati (it. galoppare), gazela (it. gazzella <ar.), granata, grandiozan (it. grandio- 
so), granit (it. granito), improvizovati (it. improvvisare), izolovati (it. isolare), kanal (it. canale), karta (it. carta), kasa (it. cassa), lava, maliciozan (it. malizioso), moto (it. motto), mozaik (it. mosaico), mumija (it. mummia), patina (it. patina), sorta, stornirati (it. stornare), torta, valuta, vila (it. villa), virtuoz (it. virtuoso).

$\mathrm{Ci}$ sono anche esempi senza adattamento morfologico delle desinenze italiane: espreso (it. espresso), fijasko (it. fiasco), finale, loto (it. lotto), neto (it. netto), solo, ecc. Difatti, gli italianismi tradizionali e quelli culturali che risalgono alle epoche precedenti al Novecento conformavano di regola le desinenze dei nomi maschili italiani (in -o e in -e) alle forme indigene serbe (apocopando la vocale finale: it. cartone $>$ karton), mentre i sostantivi di genere femminile in -a conservavano la loro desinenza anche nel passaggio in serbo (it. grottesca > s. groteska).

\section{Terminologia musicale}

A partire dal Seicento, la grande fortuna della musica e in particolare della lirica italiana è stata decisiva per la presenza attiva e il prestigio dell' $i$ taliano fuori d'Italia: l'italiano dei libretti d'opera diventa lingua universale del mondo musicale internazionale. L'italiano si conservò solo come metalingua dell'esecuzione musicale, ma il mito del suo carattere melodico sopravvisse assai più a lungo. $\mathrm{Ne}$ deriva una ricca terminologia musicale le cui componenti sono in alcuni casi passate dal filtro tedesco o quello francese (it. duetto > ted. Duett > s. duet; it. concerto > ted. Konzert > s. koncert): adađo (it. adagio), akord (it. accordo), alegro (it. allegro), andante (it. andante), arija (it. aria), bariton (fr. bariton, it. baritono), bas (fr. basse, it. basso), belkanto (it. belcanto), čembalo (it. cembalo), falset (it. falsetto), finale (it. finale), fortisimo (it. fortissimo), fuga (it. fuga), kantata (it. cantata), klarinet (it. clarinetto), krěsendo (it. crescendo), intermeco (it. intermezzo), libreto (it. libretto), maestro (it. maestro), mandolina (it. mandolino), mecosopran (it. mezzosoprano), opera (it. opera), opereta (it. operetta), partitura (it. partitura), picikato (it. pizzicato), primadona (it. primadonna), rečitativ (it. recitativo), solo (it. solo), sonata (it. sonata), sopran (it. soprano), stakato (it. staccato), tenor (it. tenore), tokata (it. toccata), trio (it. trio), trombone (it. trombone), viola (it. viola), violončelo (it. violoncello), ecc.

\section{Italogallicismi}

Come abbiamo già osservato, la cultura italiana occupò una posizione centrale in Europa fino a tutto il Cinquecento ed era patrimonio necessario degli intellettuali fino a tutto il Settecento. Durante questo dominio culturale, artistico, finanziario e commerciale una cospicua quantità di parole è passata dall'italiano in molte lingue europee, in primo luogo in francese, dal quale sono arrivate anche in serbo: ambasada (<fr. ambassade $<$ it. ambasciata), alarm (< fr. alarme < it. allarme), arkada (fr. arcade < it. arcata), balon (<fr. ballon < it. pallone), barikada (<fr. barricade < it. barricata), bataljon (< fr. bataillon < it. battaglione), bilten (< fr. bulletin < it. bollettino), brigada ( $<\mathrm{fr}$. brigade $<$ it. brigata), bravura ( $<\mathrm{fr}$. bravoure $<$ it. bravura), fasada (< fr.façade < it. facciata), freska (< fr. fresque < it. affresco, a fresco, fresco), galantan (< fr. galant < it. galante), kavalkada (< fr. cavalcade < it. cavalcata), kostim (< fr. costume $<$ it. costume), marinada (< fr. marinade $<$ it. marinata), maskarada ( $<$ fr. mascarade $<$ it. mascherata), medaljon ( $<$ fr. médaillon $<$ it. medaglione), parada (< fr.parade < it. parata), paravan (<fr.paravent $<$ it. paravento), perika $(<\mathrm{fr}$. perruque $<$ it. parrucca), pijedestal ( $<$ fr. piédestal $<$ it. piedestallo), profil (< fr.profil < it. profilo), salon (< fr. salon $<$ it. salone), serenada (< fr. sérénade < it. serenata), šarlatan ( $<$ fr. charlatan $<$ it. ciarlatano), tira$d a(<$ fr. tirade < it. tirata), tribina $(<$ fr. tribune $<$ it. tribuna), ecc. Ancora nel diciottesimo secolo l'italiano era vastamente noto dall'Europa colta ed era la seconda lingua dell'impero austroungarico (Musić 1972, 40).

\section{Nuovi italianismi in serbo}

Un recente afflusso di italianismi si è verificato negli anni sessanta e settanta del Novecento. Questa volta le motivazioni della nuova ondata di parole provenienti dalla Penisola sono piutto- 
sto diverse. A partire dagli anni sessanta, quando l'ex Jugoslavia si è aperta verso l'Occidente e quando le persone hanno cominciato a cambiare dimora per motivi di lavoro e a viaggiare per conoscere i paesi occidentali, anche la lingua italiana ha avuto un ruolo importante nell'arricchimento lessicale del serbo. Una nuova Italia del dopoguerra, sulle ali del successo cinematografico e con la modesta, ma sognatissima Cinquecento (o magari una Vespa), ha fatto sì che i giovani belgradesi degli anni sessanta-settanta invece di dire "idem po hleb" ("vado a comprare del pane"), dicevano “idem po panju”. È un esempio ormai caduto in disuso, ma possiamo ricordare il verbo kapirati (< it. capire) rimasto vivissimo nel parlato di tutte le generazioni. Inoltre: "da napravimo dir!" ("facciamo un giro?", con il verbo "napraviti” ricalcato sull' italiano fare). E i giovani di oggi comunemente dicono "Điram po gradu" ("Giro per la città").

\section{Presenza dell' italiano in vari settori}

Ma sono solo i primi segnali di un fortissimo afflusso che ha cominciato a verificarsi a partire dagli anni Novanta e che è ancora in atto. Il fenomeno riguarda vari settori: commercio, sport, gastronomia, moda e pubblicità. Grazie alla presenza, diretta o trasmessa, degli italiani nella vita degli abitanti dei pasi della ex Jugoslavia, finora abbiamo registrato una lunga serie di prestiti italiani di data recente.

\section{Industria, commercio e made in Italy}

Vi è un aspetto meno legato alla grande tradizione culturale del passato che, una trentina d'anni $\mathrm{fa}$, ha anche spinto allo studio più diffuso d'italiano: l'Italia era uno tra i dieci paesi più industrializzati del mondo per cui, in ambito internazionale, svolgeva un ruolo di primo piano sia dal punto di vista della produzione (esportazione nei settori dell'abbigliamento, del mobilio, dell'alimentazione, e prodotti di alto livello tecnologico riguardanti la chimica fine, gli strumenti scientifici di precisione, le materie plastiche, le macchine utensili), sia da quello del consumo. Lo studio della lingua italiana veniva affrontato anche in relazione ad un suo impiego per scopi pratici: soprattutto in alcune aree geografiche le percentuali di coloro che apprendono l'italiano per motivi di lavoro sono tutt'altro che trascurabili. L'idea che, dal Rinascimento in poi, connette in qualche modo l'Italia e l'italiano al bello è tutt'ora molto viva nell' immaginario collettivo: le tradizionali definizioni d'Italia come il paese dell'arte per eccellenza hanno trovato negli ultimi decenni una seria rispondenza nel grande successo del made in Italy. Tutto ciò ha un suo corrispettivo anche sul piano della diffusione della lingua italiana al di fuori d'Italia: l'italiano appare infatti legato ad uno stile di vita che si collega ad una mutata immagine dell'Italia all'estero. I prodotti made in Italy rispecchiano il profondo mutamento avvenuto negli ultimi decenni nella realtà economico-sociale d'Italia ed esportano un'idea dell'Italia ricca di prestigio. Così anche le strade centrali di Belgrado portano le insegne con nomi italiani e la moda italiana si è imposta come la più sofisticata ed elegante, mentre il design italiano è il più ricercato ed imitato (dai mobili alle automobili). Ecco alcuni nomi italiani per negozi, bar e ristoranti: Ultima moda, Azzurro, Cuoco, Mamma mia, Da Luca, Destino, Gallo nero, Giardino, Buongiorno, Casa, Castello, Pomodoro, Panefino, Primo piatto, Senza pari, Senza paura, Pappagallo, Ottimo, Bella Napoli, Tavolino, Bambino, Da Totò, Uno e tanti altri. Inoltre, altri - pseudoitalianismi - (consapevolmente o meno) imitano parole italiane: $\mathrm{Fa}$ sta pasta, Botako, kafučino, pancerota, ecc.

\section{Gastronomia}

L'enogastronomia è tradizionalmente uno dei settori di diffusione dell'italiano all'estero. Del lessico serbo fanno oramai parte i vocaboli che indicano i nomi dei famosi piatti e prodotti gastronomici italiani (pizza > pica, spaghetti > špagete/i, pasta $>$ pasta, bucatini $>$ bukatini, calamari > kalamari, carpaccio > karpačo, cassata $>$ kasato, gnocchi $>$ njoki, gorgonzola $>$ gorgonzola, mascarpone $>$ maskarpone, mozzarella $>$ mocarela, maccheroni $>$ makaroni, pesto $>$ pesto, bruschette $>$ bruskete, panettone $>$ panetone, 
risotto $>$ rižot, tagliatelle $>$ taljatele, tortellini > tortelini, lasagne $>$ lazanje, frutti di mare $>$ fruti di mare, tiramisù $>$ tiramisu) nonché bevande calde a base di caffè (espresso > espreso, cappuccino $>$ kapučino, macchiato $>$ makijato, ecc.). Pure i nomi di alcune piante e ortaggi risalgono direttamente all'italiano: origano > origano, rucola > rukola, capperi > kapari, broccoli > brokoli, pelati $>$ pelati. In questo gruppo rientrano anche molti famosi marchionimi di successo (Caffarelli 2016, 30): Barilla, Campari, Chianti, Ferrero, Galbani, Lavazza, Martini, Negroni, Nutella, Raffaello ecc.

\section{Moda}

Oltre alla gastronomia e all'automobilismo, il settore della moda si associa direttamente al fenomeno made in Italy, "ovvero quella miscela di gusto estetico, competenza artigianale e qualità delle materie prime per cui il nostro Paese è noto e apprezzato in tutto il mondo" (Sergio 2016, 55). Questo successo però si riflette piuttosto sul commercio o finanze, ma dal punto di vista linguistico "oggi la moda parla (quasi sempre) inglese" (Coveri e Fiori 2016, 69). Parlando degli italianismi di data recente, ricordiamo solo una lunga serie di marchionimi: Benetton, Bulgari, Dolce \& Gabbana, Ermenegildo Zegna, Fendi, Gianfranco Ferré, Giorgio Armani, Gucci, Max Mara, Moschino, Salvatore Ferragamo, Valentino, Versace, ecc.

\section{Sport}

Anche nell'ambito sportivo negli ultimi decenni si sono infiltrati alcuni italianismi, particolarmente nella sfera del calcio. Il mercato delle scommesse segue non solo le partite internazionali della Champions League ma anche il campionato italiano, le serie A e B. Grossomodo lo stesso discorso vale per alcuni altri sport in cui giocano anche gli atleti serbi (pallavolo, pallanuoto, pallacanestro). Ne derivano alcuni prestiti di data più o meno recente: azzurri, libero, calcio, scudetto, serie A, tifosi, giallo-rossi, rossoneri ecc.

\section{Grafia, pronuncia e morfologia lessicale}

\section{Grafia e pronuncia}

Diversamente dall'italiano, l'ortografia serba è un'ortografia fonetica. Perciò nella trascrizione dei nomi italiani praticamente si effettua la trascrizione fonetica. Il principio etimologico si mantiene solo nei prestiti integrali (Klajn 1979). Molti dei nuovi italianismi che abbiamo citato si sono impiantati nella varietà standard e hanno subito pochi adattamenti morfologici delle desinenze il che, come abbiamo già ricordato, è una novità rispetto agli italianismi tradizionali. In molti casi difatti si realizza solo la trascrizione dei nomi italiani con pochi o addirittura senza consueti adattamenti morfologici: pica, špageti, pasta, pesto, bruskete, rižoto, taljatele, tortelini, lazanje, pelati, origano, fruti di mare, nutela, espreso, kapučino, makijato, karpačo, rukola, kapari, brokoli. Quanto all'adattamento fonetico, la disposizione delle vocali e consonanti nelle due lingue è quasi analoga di modo che non sono necessari gli adattamenti in merito. Però gli ultimi fenomeni linguistici riguardo alle lingue straniere mostrano inclinazioni ad assumere gli esotismi in forma integrale, come avviene, per esempio, già da tempo con la lingua italiana nei confronti dei prestiti inglesi.

Sono interessanti e certe volte divertenti i problemi e le soluzioni di trascrizione e pronuncia dei nuovi italianismi: it. Lancia (s. Lančija, pronunciata con la $i$ che non è un segno grafico ma una vera vocale), it. Lamborghini (s. Lambordžini, come se la forma italiana fosse *lamborgini, per l'evidente influsso inglese), it. Versace (s. Versači, la $i$ per l'analogia con altre forme di cognomi uscenti in -i), it. Margherita (s. Margarita, per l'influsso del nome serbo), it. Chicco (s. Ciko, come se in italiano mancasse l'acca).

Il maggior numero degli errori si rileva negli italianismi non adattati, presenti nella gastronomia, soprattutto nei menù dei ristoranti (i quali spesso riportano nella lingua d'origine - ma con moltissimi errori - i nomi dei piatti italiani). È un uso, diciamo, ancora fuori controllo, non istituzionalizzato, che avviene nel tentativo 
di dare un'impronta originale al pasto da consumare. Considerato che il serbo non conosce le geminate, in molti esempi le stesse mancano, o si aggiungono là dove non dovrebbero stare, oppure c'è un "misto" di trascrizione e forma originale: *mozzarela, *nuttela, ${ }^{*}$ capriciosa, *quatro stagione, * quatro formagi, *fruti di mare, *meditteranea, *calzona, *fungi, *tono, *lazagna, ${ }^{*}$ pesto genoveze, ${ }^{*}$ bologneze, ${ }^{*}$ sicilijana e addirittura ${ }^{*}$ serbijana.

\section{Morfologia lessicale}

Suffissi italiani-radici serbe

Il fenomeno più sorprendente è l'influsso sulla morfologia lessicale, in particolare sui suffissi derivazionali. Oltre ai suffissi latini e greci passati attraverso l'italiano (-ante, -ente, -ario, -ese, -ista, -ite, - tore, -izzare), a partire dalla fine degli anni novanta si registra un notevole aumento di applicazione di suffissi alterativi italiani alle basi serbe o altre: jogurtino, tomatello, tomatino, sizela, smirela, ecc. Il fenomeno è presente soprattutto nel linguaggio della pubblicità, precisamente nei marchi commerciali di alcuni prodotti (tomatello, tomatino, kremissimo) oppure nel linguaggio giovanile o nel parlato informale (laganini, laganese). Potremmo definire questi esempi come pseudoitalianismi che comunque dimostrano la fortissima influenza della lingua italiana sul serbo.

\section{Desinenze serbe / suffissi serbi - radici italiane}

Questo fenomeno è presente anzitutto nelle forme verbali in cui la radice è italiana e le desinenze appartengono invece alla morfologia verbale serba: it. girare > s. dirati (diram, diraš, dira...), it. capire > s. kapirati (kapiram, kapiras, kapira...). Un caso interessante che illustra parallelismi fra le due lingue è l'esempio di trippa che come prestito italiano si usa con il suffisso diminutivo slavo -ica, tripica, precisamente tripice (in uso è la forma plurale). In serbo si usa la parola indigena, sempre nella forma diminutiva e plurale per indicare questo piatto caratteristico dei mangioni (škembići).

\section{Considerazione conclusiva}

Le parole serbe diventano italiane? Nei dizionari italiani, al contrario, vengono registrate pochissime voci di origine serba. Si tratta o di parole che indicano rappresentanti di varie guerriglie locali o di nomenclature nobiliari del passato (uscocco, voivoda, vladika, bano, cetnico, ustascia ecc.) o di prodotti tipici dei Balcani (paprica, gusla, rachi). Inoltre, nel lessico italiano vi sono le parole stravizzo e stravizio, che derivano dal serbo $z$ dravica (brindisi). Si tratta di un vocabolo giunto in Italia per tramite veneziano. Il primo esempio risale addirittura al 1473 (cfr. Cortelazzo e Zolli 2004).

Da quanto è stato affermato, si può concludere che il contatto fra le due lingue è unidirezionale. Anche se i pochissimi esempi lo potrebbero negare, non offrono tuttavia nuove prove che contestino l'affermazione che l'italiano è sempre stato lingua di prestigio rispetto al serbo e che così sarà anche nel futuro. Del resto, una situazione analoga si verifica anche in riferimento ad altre lingue slave che, nel passato, hanno esercitato sull'italiano un' influenza molto modesta e limitata alle nozioni locali e nazionali (bolscevico, polca, zar, kolkhoz, astrakan, bortsch, tundra, glasnost, perestroika). Così il serbo per l'italiano è solo una lingua esotica poiché gli slavismi in italiano si riferiscono solo ed esclusivamente alle nozioni tipiche della cultura nazionale.

\section{Summary}

In this article, the author confirms that Italian, as a great language of culture, in various periods has had a significant influence on the Serbian lexicon. The paper offers a diachronic review of the influences that Italian has exerted on the Serbian: due to the close and different contacts between the two sides of the Adriatic that have lasted for centuries, the Serbian language contains a large number of italianisms. On the other hand, the article confirms the hypothesis that only very few Serbian words entered Italian. The author concludes that the relationship between the two languages is one-way and that Italian is a prestigious language compared to Serbian. 


\section{Povzetek}

V prispevku avtorica izpostavlja, da je italijanščina, kot jezik velike kulture, v različnih obdobjih pomembno vplivala na bogatenje srbskega besedišča. Prispevek predstavi diahroni pregled vplivov, ki jih je italijanščina imela nad srbskim jezikom: zaradi tesnih in raznolikih večstoletnih stikov med dvema jadranskima obalama, srbščina vsebuje veliko število italijanizmov. Članek obenem potrjuje dejstvo, da je samo nekaj srbskih besed zaživelo v italijanskem jeziku. Avtorica izpostavlja, da je odnos med dvema jezikom enosmeren in da je italijan-

\section{Riferimenti bibliografici}

AA.VV. 2007. Rečniksrpskogjezika. Novi Sad: Matica Srpska.

Arcangeli, M. 2007. "Il lessico sportivo e ricreativo italiano nelle quattro grandi lingue europee (con qualche incursione anche altrove)." Studi di lessicografia italiana XXIV: 195-248.

Berruto, G. 1995. Fondamenti di sociolinguistica. Roma-Bari: Laterza.

Bertini Malgarini, P. 1994. "L'italiano fuori d'Italia.” In Storia della lingua italiana. Volume terzo: le altre lingue, a cura di Luca Serianni e Pietro Trifone, 883-922. Torino: Einaudi.

Bettoni, C. 1993. "Italiano fuori d'Italia." In Introduzione all'italiano contemporaneo. La variazione e gli usi, a cura di Alberto A. Sobrero, 4II-460. Roma-Bari: Laterza.

Caffarelli, E. 2016. "I marchionimi italiani e la loro diffusione internazionale." In L'italiano e la creatività: marchie costumi, moda e design (La lingua italiana nel mondo), a cura di Paolo D'Achille e Giuseppe Patota, 29-48. Firenze: Accademia della Crusca.

Casapullo, R. 2009. “Italiano fuori d'Italia: una panoramica sul lessico italiano della scienza nelle lingue europee." Annali dell'Università Suor Orsola Benincasa": 637-85.

Cortelazzo, M. e P. Zolli. 2004. Dizionario etimologico della lingua italiana. Bologna: Zanichelli.

Coveri, L. e F. Fiori. 2016. "La moda parla (ancora) italiano?” In L'italiano e la creativita: marchi e costumi, moda e design (La lingua italiana nel mondo), a cura di Paolo D’Achille e Giuseppe Patota, 69-74. Firenze: Accademia della Crusca.

D'Achille, P. e G. Patota. 2016. L'italiano e la creatività: marchi e costumi, moda e design (La lingua italiana nel mondo). Firenze: Accademia della Crusca.

Klajn, I. 1979. "Transkripcija i adaptacija imena iz romanskih jezika.” In Radovi VI: I2 II74.

Klajn, I. 1996. "Vrste romanizama u savremenom srpskohrvatskom jeziku i putevi njihovog dolaska." Zbornik Matice srpske za filologiju i lingvistiku XXIX/2: 45-64.

Klajn, I. e M. Šipka. 2008. Veliki rečnik stranib reči i izraza. Novi Sad: Prometej.

Lipovac-Radulović, V. 1981. Romanizmi u Crnoj Gori - Jugoistočni dio Boke Kotorske. Cetinje: Obod.

Lipovac-Radulović, V. 2009. Romanismi lessicali in Montenegro: Budua e Pastrovici: la parte sud-orientale delle Bocche di Cattaro. Roma: Il Calamo.

Musić, S. 1972. Romanizmi u severozapadnojBoki Kotorskoj. Beograd: Filološki fakultet.

Rossi, L. e R. Wank. 2oro. "La diffusione dell' italiano nel mondo attraverso la religione e la Chiesa cattolica: ricerche e nuove prospettive." In L'italiano nella Chiesa fra passato e presente, a cura di Massimo Arcangeli, II3-I7I. TorinoLondra-Venezia-New York: Allemandi. Sergio, G. 2016. "Italianismi di moda nelle lingue del mondo.” In L'italiano e la creatività: marchi e costumi, moda e design (La lingua italiana nel mondo), a cura di Paolo D'Achille e Giuseppe Patota, 55-68. Firenze: Accademia della Crusca. 
Serianni, L. 2017. "L'italiano nel mondo. Intenti e propositi di un progetto editoriale sugli italianismi”. In Osservatorio degli italianisminel mondo. Punti di partenza e nuovi orizzonti, a cura di Matthias Heinz, 39-54. Firenze: Accademia della Crusca.

Stammerjohanm, H. 20I0. "Italianismi."

In Enciclopedia Treccani http://www. treccani.it/enciclopedia/italianismi (Enciclopedia-dell'Italiano)/

Zolli, P. 1976. Le parole straniere. Bologna: Zanichelli.

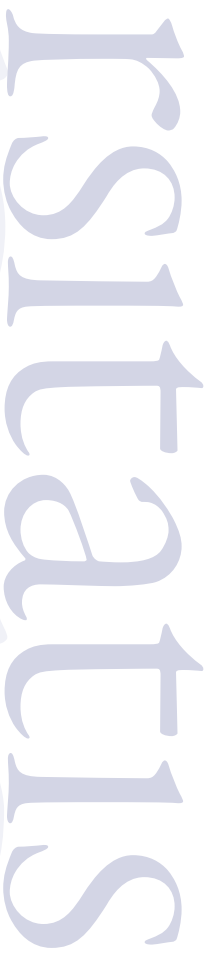

